\title{
Migración Interna en Cuatro Ciudades de Honduras ${ }^{1}$
}

\author{
Manuel Antonio Flores Fonseca ${ }^{2}$
}

\section{Resumen}

Este estudio se enfoca en cuatro ciudades, que son las principales desde el punto de vista económico (la capital gubernamental, la primera y segunda industrial), demográfico al ser las más pobladas y de mayor crecimiento y desde el punto de vista migratorio porque tienen los mayores saldos positivos del país en la migración de toda la vida y reciente. Mantienen su atractivo migratorio reciente y la cuantía desde el resto del país continúa siendo una fuerza importante. En ellas la migración neta positiva se debe exclusivamente al intercambio migratorio con otros municipios ubicados en otras DAM del resto de país, pues la migración neta con otros municipios de su DAM es menor. En San Pedro Sula el saldo migratorio positivo resulta de la diferencia que es mayor de la combinación del intercambio con los municipios pertenecientes a las otras DAM y el saldo negativo en el intercambio con el resto de municipios de su propia DAM, con lo que este municipio tiene la tendencia de pérdida de atractivo en su DAM producto posiblemente a la ampliación de sus límites a los municipios adyacentes, que incluye a los municipios de Choloma, Villanueva y otros vecinos que pertenecen a otra DAM.

\begin{abstract}
This research is focused on four major cities, major from an economic point of view (the capital and the first and second industrial cities), from a demographic point of view since these are the most populated areas, and from a migration perspective because they have the largest positive balance of the country in all-life or recent migration. They maintain their recent migratory attraction and the amount of the rest of the country is still an important movement. In them, the net positive migration is due exclusively by the migratory exchange between municipios located in other DAM from the rest of the country, since net migration with other municipios is less. In San Pedro Sula, the positive balance in results from the diference that is greater than the exchange combination with municipios from other DAM and the negative saldo from the exchange with the rest of the municipios from their own DAM, with which this municipio the tendency of losing attraction in its DAM possibly because of the expansion of its limits to adjacent municipios, this includes the municipios of Choloma, Villanueva, and other neighbors that belong to another DAM.
\end{abstract}

Palabras clave: Migración; Migración interna; DAME; migration; internal migration. 


\section{INTRODUCCIÓN}

Honduras presenta un nivel de baja movilidad interna a nivel de DAM y DAME según los datos de los dos últimos censos (1988 y 2001) al compararlos con los censos de los demás países de América Latina, tanto en

Volumen I

$\mathbf{N}^{\circ} 2$

2010 la migración absoluta como en la reciente, aún cuando la extensión territorial es pequeña y la cantidad de DAM y DAME es elevada. Esta movilidad no despreciable, es reflejo de las desigualdades económicas regionales y locales fortalecidas por el carácter de los departamentos, municipios y ciudades, tal es el caso del gubernamental en Tegucigalpa, industrial en San Pedro Sula y más recientemente por el boom maquilero en ciudades de Choloma y Villanueva. Estimaciones de migración rural-urbana apuntan que la migración es todavía parte importante del crecimiento urbano, sin embargo, la mayoría de los movimientos migratorios son urbanos-urbanos (Flores. 2006: 22).

Este artículo está estructurado en cuatro secciones, de las cuales la primera incluye los aspectos introductorios, objetivo, planteamiento del problema, fuentes de información y metodología. La sección dos incluye un panorama general de la migración interna de Honduras en lo relativo a cuantía, volumen, saldos migratorios y principales flujos migratorios a nivel de DAM y DAME en la migración absoluta y la reciente. La sección tres recoge la justificación del estudio enfocado en cuatro ciudades, los aspectos históricos, demográficos, los estrictamente migratorios internos como ser magnitud, cuantía, saldos migratorios, flujos migratorios y la migración cercana y lejana en las cuatro ciudades. La sección cuatro presenta algunos elementos finales a manera de conclusión.

\subsection{Objetivo del trabajo.}

El objetivo de esta investigación es llenar los vacíos de información de migración interna, específicamente conocer el atractivo migratorio reciente y otros efectos de la migración en cuatro municipios donde se ubican ciudades importantes de Honduras, siendo Tegucigalpa, San Pedro Sula, Choloma y Villanueva.

\subsection{Planteamiento del problema.}

El estudio de la migración interna en América Latina aunque data desde los años setenta cuando se profundizó su estudio a raíz del interés por los desplazamientos del campo a las ciudades, en los últimos años se ha potenciado con nuevos instrumentos que ha permitido que los estudios regionales nos ilustren exhaustivamente sobre la situación de esta importante variable. El Centro Latinoamericano de Demografía (CELADE) ha jugado un papel importante en la región, ha desarrollado software de optimización de datos censales, programas de capacitación sobre la temática, puesto a disponibilidad las matrices de migración de los países de la región y los estudios regionales que clarifican importantes hallazgos y abordan los aspectos metodológicos para el análisis de la migración interna ${ }^{4}$.

En ese bagaje de información sobre migración interna un aspecto que ha cobrado vigencia es el estudio de la migración hacia áreas metropolitanas en la región, encontrando evidencias de la relevancia socioeconómica y política de estos aglomerados, su enorme peso demográfico en los sistemas de asentamientos nacionales, la evidencia entre 1950 y 1970 de flujos migratorios hacia área metropolitanas ingentes y sostenidos, y en los años ochenta alguna áreas metropolitanas emblemáticas perdieron atractivo para los

3 DAM es la división administrativa mayor y DAME es la división administrativa menor, en este caso es el departamento y el municipio respectivamente

4 CELADE ha desarrollado el programa REDATAM utilizado en el procesamiento de información censal entre esta, la de migración. Desarrolló el programa Migración Interna en América Latina y el Caribe MIALC con la implementación de bases de datos sobre la temática, Un programa de capacitación sobre migración interna en varios países de la región y varios estudios regionales sobre migración. 
migrantes, mientras los hallazgos de los años noventa mostraban metrópolis de crecimiento aún vigoroso y de pertinaz atractivo para los migrantes y algunos de los conteos también evidenciaron la recuperación del crecimiento demográfico de las metrópolis y revalorización de las ciudades globales (Rodríguez, 2004: 22).

En los estudios regionales de migración interna reciente en algunas áreas metropolitanas se encontró que el factor distintivo clave de la cuantía demográfica de las ciudades, las más pobladas como Ciudad de México, Río de Janeiro, Sao Paulo y Santiago tenían emigración neta, mientras las de menor tamaño demográfico registraban todavía una inmigración neta, validando la hipótesis de la pérdida de atractivo de las metrópolis a la vez se reconocían sus límites. Para un grupo importante de ciudades, menores de cuantía demográfica pero principales en el sistema de ciudades el atractivo migratorio seguía operando y la migración desde el resto del país continuaba siendo una poderosa fuerza de cambio. Más recientemente se ampliaron nuevos datos y las técnicas, entre ellas las estimaciones de efectos netos y exclusivo de la migración en los lugares de origen y destino, asimismo en la elaboración de la información es posible hacer distinciones entre los flujos de migración interna cercana, que es el intercambio con municipios fuera del aglomerado metropolitano pero dentro de la DAM donde se localiza el aglomerado, y la migración interna lejana, que es el intercambio con municipios fuera de la DAM donde se localiza el aglomerado (Rodríguez y Busso, 2009: 121).

Utilizando esta técnica pretendemos conocer a que se debe la migración interna neta positiva de los municipios donde se localizan las ciudades de Tegucigalpa en el centro, San Pedro Sula, Choloma y ViIlanueva en el Valle de Sula en el norte de Honduras.

\subsection{Fuentes de información.}

La principal fuente de datos de esta investigación es el Censo de Población y Vivienda del 2001. Se utilizan las matrices de migración básicas, de toda la vida y reciente a nivel de DAM y DAME generadas de la base censal y las construidas por el programa MIALC del Centro Latinoamericano de Demografía (CELADE). Con estas matrices se estiman los efectos de la migración en los lugares de origen y destino y específicamente los efectos de los flujos migratorios cercanos y lejanos en las cuatro ciudades objeto de estudio.

\subsection{Metodología.}

La metodología utilizada en este estudio descriptivo fue construir a partir de la información recogida en las fuentes de datos secundarias, las series de información necesarias que permitieran conocer las principales tendencias del pasado y presente del comportamiento de las variables a utilizar.

\section{MIGRACIÓN INTERNA EN HONDURAS.}

A manera de contextualización se presenta un panorama migratorio del país en los últimos años, que sin duda ilustra la cuantía de migrantes, su evolución a través del tiempo, los saldos migratorios y los principales flujos migratorios, tanto en la migración de toda la vida como la reciente a nivel de DAM y DAME. 


\subsection{Cuantía y magnitud.}

A nivel de país se han hecho estimaciones de migración interna a nivel de DAM y se ha encontrado que la proporción de migrantes internos absolutos residente en un departamento diferente al de su nacimiento fue de un continuo aumento entre 1950 y 1974, cambiando esta tendencia al descenso desde ese año. En

Volumen I

$\mathbf{N}^{\circ} \mathbf{2}$

2010 el 2001 se estima que esta proporción alcanza el 17.2\% (Cuadro No.1). A nivel de DAME la migración interna de toda la vida ha aumentado en términos absolutos entre el período 1988 - 2001 en más de un cuarto del millón de personas, sin embargo, en términos relativos ha significado una reducción de un cuatro por ciento, de $27.5 \%$ a $23.3 \%$ (Flores, 2009: 256).

\begin{tabular}{|c|c|c|c|c|}
\hline & Año censal & $\begin{array}{l}\text { Número de migrantes } \\
\text { absoluto entre DAM }\end{array}$ & $\begin{array}{c}\text { Población censal rele- } \\
\text { vante }\end{array}$ & $\begin{array}{l}\text { Proporción de migran- } \\
\text { tes internos absolutos } \\
\text { entre DAM (por cien) }\end{array}$ \\
\hline & 1950 & 112,840 & $1,335,741$ & 8.45 \\
\hline & 1961 & 277,574 & $1,833,561$ & 15.14 \\
\hline & $1974^{*}$ & 513,513 & $2,656,948$ & 19.33 \\
\hline & 1988 & 809,025 & $4,140,511$ & 19.54 \\
\hline & 2001 & $1,040,883$ & $6,053,955$ & 17.20 \\
\hline & Año censal & $\begin{array}{l}\text { Número de migrantes } \\
\text { absolutos entre DAME }\end{array}$ & $\begin{array}{c}\text { Población censal rele- } \\
\text { vante }\end{array}$ & $\begin{array}{l}\text { Proporción de migran- } \\
\text { tes internos absolutos } \\
\text { entre DAME (por cien) }\end{array}$ \\
\hline & $1950 * *$ & & & \\
\hline & $1961 * *$ & & & \\
\hline & $1974 * * *$ & & & \\
\hline \multirow[t]{2}{*}{ Pág 153} & 1988 & $1,130,464$ & $4,112,367$ & 27.49 \\
\hline & 2001 & $1,390,823$ & $5,963,794$ & 23.32 \\
\hline
\end{tabular}

Cuadro No.1 Honduras: Cuantía y magnitud relativa de la Migración Absoluta entre DAM y DAME según Censo de Población.

*En el censo de población de 1974, la población relevante es la total, ya que no fueron deducidos los nacidos en el exterior, que se presume alcanzaban un número significativo, por lo que en este año se registró la proporción más alta de migrantes a nivel de la división administrativa mayor.

** No se formuló la pregunta sobre lugar de nacimiento a nivel de división administrativa menor.

*** No se conocen tabulaciones a nivel de la división administrativa menor.

Fuente: Elaboración propia, sobre la base de Centro Latinoamericano y Caribeño de Demografía (CELADE) - División de Población de la CEPAL, base de datos de Migración Interna en América Latina y el Caribe (MIALC).

La migración interna reciente a nivel de DAM en Honduras en las últimas tres décadas tiene la tendencia a la disminución de su proporción. Lo mismo sucede con su intensidad a nivel de DAME que aunque aumentó en términos absolutos entre las mediciones del período 1988 - 1983 y 2001 - 1996 en más de ochenta mil personas, en términos relativos ha significado una reducción de uno y medio por ciento del $13.7 \%$ a 12.1\% (Cuadro No.2). 


\begin{tabular}{|c|r|r|r|r|}
\hline $\begin{array}{c}\text { Año } \\
\text { censal }\end{array}$ & $\begin{array}{c}\text { Número de migran- } \\
\text { tes absoluto entre } \\
\text { DAM }\end{array}$ & $\begin{array}{c}\text { Población } \\
\text { censal rele- } \\
\text { vante }\end{array}$ & $\begin{array}{c}\text { Proporción de migran- } \\
\text { tes internos absolutos } \\
\text { entre DAM (por cien) }\end{array}$ & $\begin{array}{c}\text { Tasa media anual de } \\
\text { migración entre DAM } \\
\text { (por mil) }\end{array}$ \\
\hline 1974 & 164,625 & $2,068,102$ & 7.96 & 15.9 \\
\hline 1988 & 167,530 & $3,404,003$ & 4.92 & 9.24 \\
\hline 2001 & 219,650 & $5,184,982$ & 8.5 \\
\hline Año & $\begin{array}{c}\text { Número de migran- } \\
\text { tes absolutos entre } \\
\text { censal }\end{array}$ & $\begin{array}{c}\text { Población } \\
\text { censal rele- } \\
\text { vante }\end{array}$ & $\begin{array}{c}\text { Proporción de migran- } \\
\text { tes internos absolutos } \\
\text { entre DAME (por cien) }\end{array}$ & $\begin{array}{c}\text { Tasa media anual de } \\
\text { migración entre DA- } \\
\text { ME(por mil) }\end{array}$ \\
\hline $1974^{* *}$ & 232,572 & $3,397,022$ & 6.85 & 13.7 \\
\hline 1988 & 312,622 & $5,166,813$ & 6.05 & 12.1 \\
\hline 2001 & & & & \\
\hline
\end{tabular}

Cuadro No.2 Honduras: Cuantía y Magnitud Relativa de la Migración Reciente entre DAM y DAME según Censo de Población*

* En los censos de población de 1950 y 1961 no se incluyó la pregunta sobre lugar de residencia cinco años antes.

** No se conocen tabulaciones a nivel de la división administrativa menor.

Fuente: Elaboración propia, sobre la base de Centro Latinoamericano y Caribeño de Demografía (CELADE) - División de Población de la CEPAL, base de datos de Migración Interna en América Latina y el Caribe (MIALC).

El proceso migratorio está asociado a la estructuración de la infraestructura socio productiva del país, donde fenómenos como la ampliación de la frontera agrícola, la ampliación de la red vial, la conformación de ciudades intermedias y el fortalecimiento de una urbanización bipolar (Tegucigalpa y San Pedro Sula) ha influido para tener un patrón migratorio hacia el corredor central y una primacía de la zona norte.

\subsection{Saldos migratorios internos.}

En el país en términos absolutos y relativos han aumentado en las dos últimas operaciones censales (1988 y 2001) las DAM y las DAME con saldo migratorio negativo, tanto en la migración de toda la vida, como en la reciente, esto demuestra mayor concentración en los flujos migratorios internos (Cuadro No.3).

\begin{tabular}{|l|r|r|r|r|r|r|r|r|}
\cline { 2 - 9 } \multicolumn{1}{c|}{} & \multicolumn{2}{c|}{$\begin{array}{c}\text { Migración de to- } \\
\text { da la vida DAM }\end{array}$} & \multicolumn{2}{c|}{$\begin{array}{c}\text { Migración reciente } \\
\text { DAM }\end{array}$} & \multicolumn{2}{c|}{$\begin{array}{c}\text { Migración de to- } \\
\text { da la vida DAME }\end{array}$} & \multicolumn{2}{c|}{$\begin{array}{c}\text { Migración reciente } \\
\text { DAME }\end{array}$} \\
\hline Saldo Migratorio & 1988 & 2001 & $1988-1983$ & $2001-1996$ & 1988 & 2001 & $1988-1983$ & $2001-1996$ \\
\hline Positivo & 6 & 5 & 7 & 4 & 80 & 74 & 73 & 70 \\
\hline Negativo & 12 & 13 & 11 & 14 & 209 & 224 & 216 & 228 \\
\hline Total & 18 & 18 & 18 & 18 & 289 & 298 & 289 & 298 \\
\hline
\end{tabular}

Cuadro No.3 Honduras: Número de DAM y DAME por tipo de migración según saldo migratorio, 1998 y 2001. Fuente: Cálculos propios en base a datos censales de 1988 y 2001.

Migración Interna en Cuatro Ciudades de Honduras 
Volumen I

$\mathbf{N}^{\circ} 2$

2010

En las últimas cinco décadas a través del análisis de las matrices de migración de toda la vida a nivel de DAM los departamentos de Cortés, Francisco Morazán, Colón, Atlántida e Islas de la Bahía generalmente han presentado un saldo migratorio positivo, con una alta concentración en los primeros dos (donde se ubican la capital industrial y la gubernamental que son San Pedro Sula y Tegucigalpa respectivamente), esto hace a estas dos DAM sean las más atractivas del país y que se ha manifestado a través del tiempo, mientras que con saldos migratorios negativos aparecen los restantes departamentos, siendo más notoria esta característica en Choluteca, Santa Bárbara, Copán, Lempira y Valle (véase el Gráfico No.1).

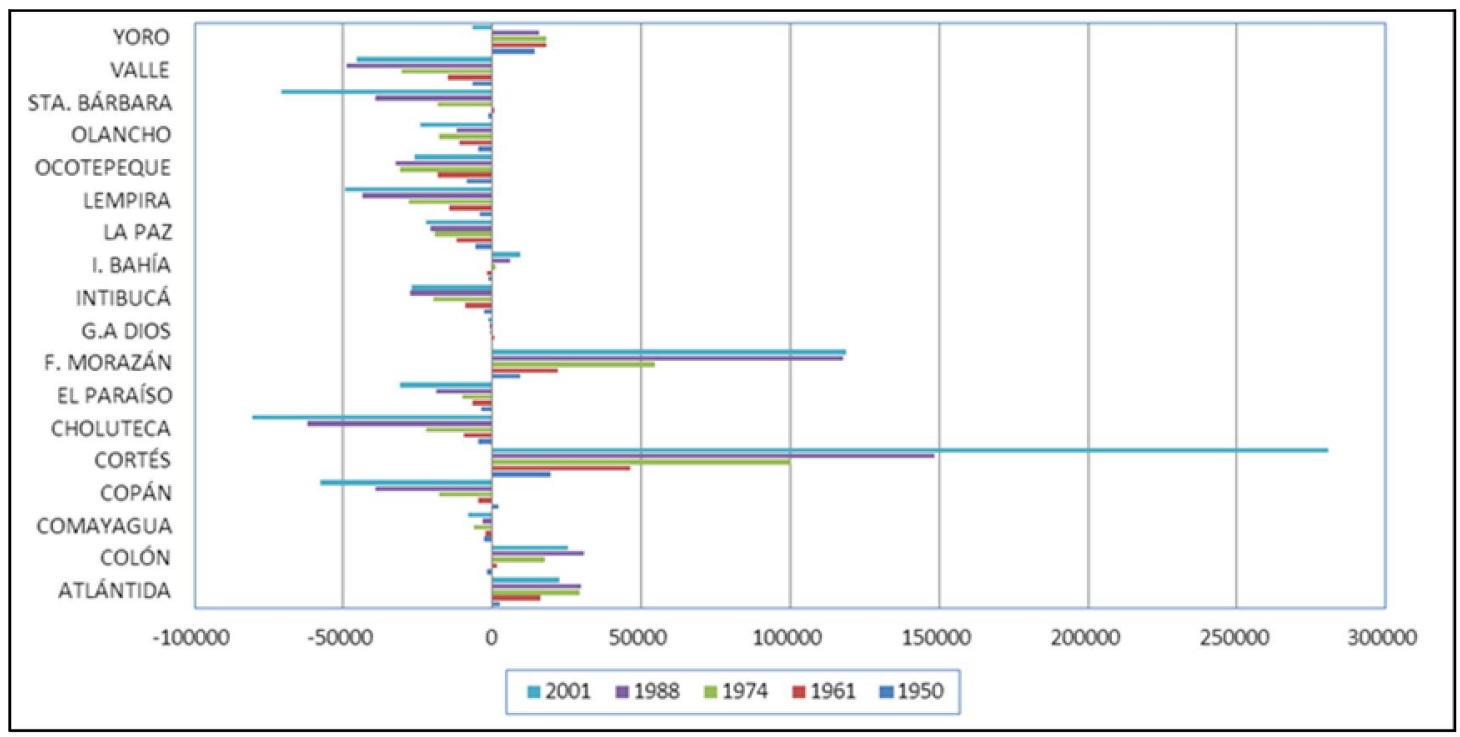

Gráfico 1. Honduras: Saldos Migratorios de toda la Vida, DAM 1950-2001

La migración interna reciente a nivel de DAM muestra también una tendencia a la concentración, donde Cortés y Francisco Morazán abarcan la mayoría de los desplazamientos recientes y en el primero la atracción es abismalmente mayor porque se suman la fuerza de atracción de fuerza de trabajo que genera la zona metropolitana del Valle de Sula ${ }^{5}$ (véase el Gráfico No.2).

Al construir la matriz de migración a nivel de DAME del censo de población del 2001 los municipios con mayor número de emigrantes internos de toda la vida son San Pedro Sula y Tegucigalpa que albergan a las dos ciudades principales del país (expulsan el $11 \%$ de los emigrantes). También son los más atractivos (atraen el $28 \%$ de los inmigrantes del país).

Los municipios con mayores saldos migratorios positivos de toda la vida son los que albergan a las dos grandes ciudades del país (Tegucigalpa y San Pedro Sula), municipios atractivos por la industria maquiladora (Choloma, Villanueva, Puerto Cortés, El Progreso), ciudades intermedias y algunos de frontera agrícola o cercanos a ella (Patuca, Trojes, Tocoa, Bonito Oriental, Catacamas), por industria turística como Roatán (Cuadro No.4).

Los municipios con mayor saldo migratorio negativo son aquellos donde la emigración es mayor que la inmigración y son generalmente municipios expulsores de población de tradición del occidente del país (Santa Bárbara, Santa Rosa de Copán, Ocotepeque, San José de Colinas), o en el litoral norte (Tela, Yoro,

5 Con urbanización situada en Valle aluvial más grande de Honduras y en la cuenca de los ríos Ulúa, Chamelecón y Humuya. Comprende un total de 7,384.6 kilómetros cuadrados, la integran 18 municipios de tres departamentos, siendo ellos, San Pedro Sula, Choloma, Puerto Cortés, La Lima, Omoa, Pimienta, Potrerillos, San Antonio de Cortés, San Francisco de Yojoa, San Manuel, Santa Cruz de Yojoa, Villanueva, El Progreso, El Negrito, Santa Rita, Quimistán, Petoa y Tela. 


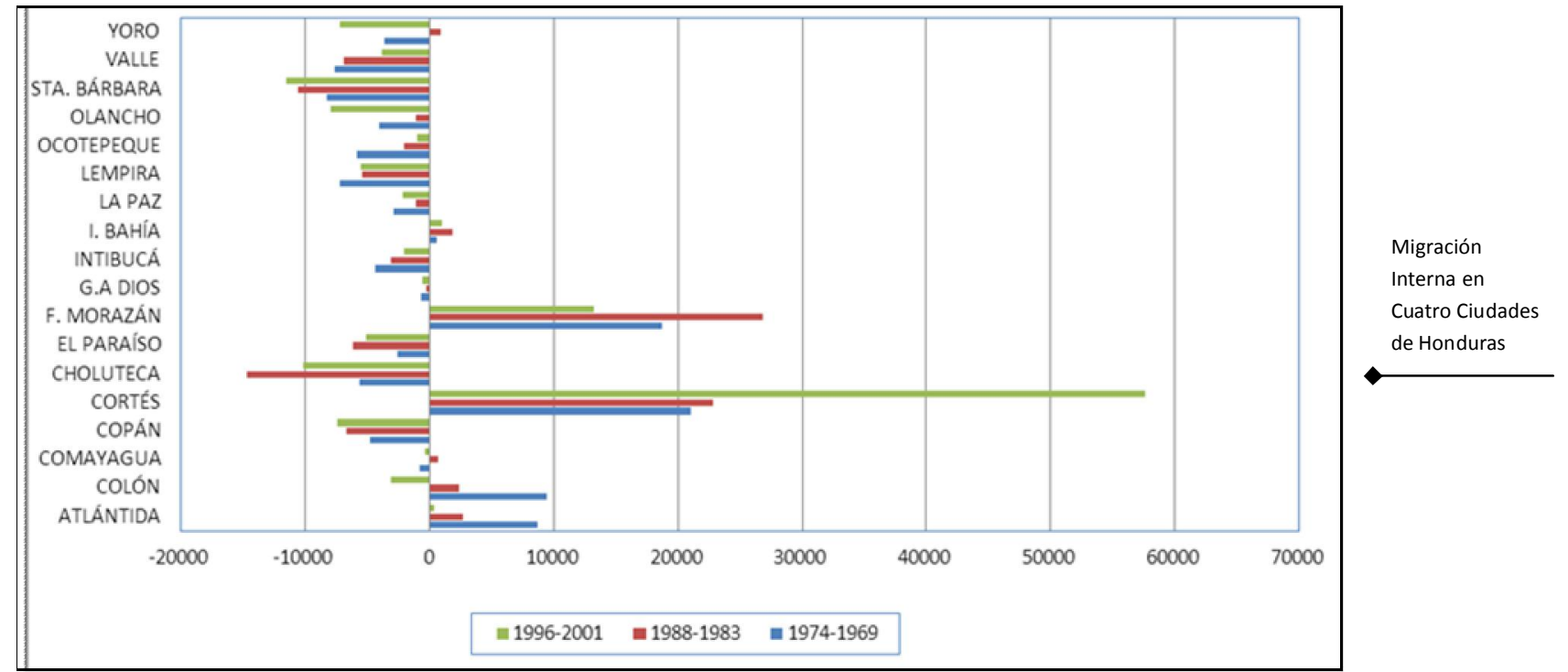

Gráfico 2. Honduras: Saldos migratorios recientes, DAM 1969-1974, 1983-1988, 1996-2001

Olanchito) generalmente hacia San Pedro Sula. Otros son municipios del área sur, oriente y oeste que generalmente expulsan población a Tegucigalpa.

\begin{tabular}{|l|l|r|}
\hline Cód. & \multicolumn{1}{|c|}{ Municipios } & $\begin{array}{c}\text { Migración Neta } \\
1988\end{array}$ \\
\hline 801 & Distrito Central (Teg) & 150,607 \\
\hline 501 & San Pedro Sula & 61,021 \\
\hline 502 & Choloma & 26,223 \\
\hline 512 & La Lima & 14,742 \\
\hline 1804 & El Progreso & 13,363 \\
\hline 506 & Puerto Cortés & 11,843 \\
\hline 103 & Esparta & 10,171 \\
\hline 719 & Trojes & 10,135 \\
\hline 511 & Villanueva & 9,150 \\
\hline 510 & Santa Cruz de Yojoa & 8,906 \\
\hline
\end{tabular}

\begin{tabular}{|l|l|r|}
\hline Cód. & \multicolumn{1}{|c|}{ Municipios } & $\begin{array}{c}\text { Migración Neta } \\
\mathbf{2 0 0 1}\end{array}$ \\
\hline 801 & Distrito Central (Teg) & 140,318 \\
\hline 501 & San Pedro Sula & 92,548 \\
\hline 502 & Choloma & 64,379 \\
\hline 511 & Villanueva & 34,590 \\
\hline 506 & Puerto Cortés & 16,199 \\
\hline 512 & La Lima & 14,571 \\
\hline 509 & San Manuel & 14,202 \\
\hline 1804 & El Progreso & 12,564 \\
\hline 510 & Santa Cruz de Yojoa & 10,688 \\
\hline 101 & La Ceiba & 10,479 \\
\hline
\end{tabular}

Cuadro 4. Honduras: Diez Municipios con mayor saldo migratorio positivo de toda la vida, 1988 y 2001. (Fuente: Cálculos propios en base censal y MIALC, CELADE.)

Los municipios con mayor saldo migratorio positivo reciente fueron Villanueva y Choloma que superaron en la primacía a Tegucigalpa y San Pedro Sula y se convirtieron en los últimas dos décadas en municipios altamente atractivos por la creciente generación de empleo en maquilas (Cuadro No.5). Los 
municipios de mayor saldo migratorio negativo reciente incluye una mayor diversificación, a excepción de Tela en el norte y Pespire, El Triunfo, Langue y Concepción de María en el sur del país que tienen una larga tradición de perder poblaciones por emigración.

Volumen I

$\mathbf{N}^{\circ} 2$

2010

\begin{tabular}{|l|l|r|}
\hline Cód. & \multicolumn{1}{|c|}{ Municipios } & $\begin{array}{r}\text { Migración Neta } \\
\text { 1988 - 1983 }\end{array}$ \\
\hline 801 & Distrito Central (Teg) & $\mathbf{3 0 , 0 3 1}$ \\
\hline 501 & San Pedro Sula & $\mathbf{8 , 1 2 0}$ \\
\hline 502 & Choloma & 6,372 \\
\hline 512 & La Lima & 2,654 \\
\hline 1804 & El Progreso & 1,841 \\
\hline 511 & Villanueva & 1,533 \\
\hline 509 & San Manuel & 1,331 \\
\hline 1101 & Roatán & 1,188 \\
\hline 1503 & Catacamas & 1,178 \\
\hline 506 & Puerto Cortés & 1,001 \\
\hline
\end{tabular}

\begin{tabular}{|l|l|r|}
\hline Cód. & \multicolumn{1}{|c|}{ Municipios } & $\begin{array}{c}\text { Migración Neta } \\
\text { 2001 - 1996 }\end{array}$ \\
\hline 502 & Choloma & $\mathbf{2 0 , 2 2 3}$ \\
\hline 511 & Villanueva & 14,361 \\
\hline 801 & Distrito Central (Teg) & 11,452 \\
\hline 501 & San Pedro Sula & 6,889 \\
\hline 509 & San Manuel & 3,922 \\
\hline 318 & Siguatepeque & 2,197 \\
\hline 506 & Puerto Cortés & 2,010 \\
\hline 504 & Pimienta & 1,822 \\
\hline 510 & Santa Cruz de Yojoa & 1,781 \\
\hline 101 & La Ceiba & 1,347 \\
\hline
\end{tabular}

Cuadro 5. Honduras: Diez municipios con mayor saldo migratorio positivo reciente, 1988-83 y 2001-96. (Fuente: Cálculos propios en base censal y MIALC, CELADE.)

A su vez el grado de urbanización y el saldo migratorio están asociados, se ha encontrado que a menor grado de urbanización los municipios tienen generalmente saldos migratorios negativos y a mayor grado de urbanización los municipios tienen saldos migratorios positivos. Otra relación encontrada en el fenómeno migratorio es que a menor grado de pobreza los municipios generalmente tienen saldos migratorios positivos y a mayor grado de pobreza los municipios generalmente tienen saldos migratorios negativos (Flores, 2005: 32). En el caso de la migración reciente también se produce el mismo fenómeno, de que a menor pobreza los saldos son generalmente positivos y a mayor pobreza el saldo generalmente es negativo, ya sea a nivel municipal y departamental. A esta relación se suma la ubicación de las principales ciudades del país, la mejor infraestructura física tanto vial, aérea y portuaria, institucional (gubernamental y no gubernamental) e industrial del país en los departamentos considerados atractivos para migrar en desmedro con las unidades geográficas deprimidas consideradas de expulsión.

\subsection{Flujos migratorios internos.}

Los principales flujos migratorios de toda la vida a nivel de DAME que son mayores de dos mil personas en el 2001 se produjeron principalmente hacia Tegucigalpa, San Pedro Sula y otras ciudades intermedias como Choloma, Villanueva, La Lima, La Ceiba, El Progreso y Puerto Cortés. Específicamente los mayores flujos se produjeron de San Pedro Sula a Choloma, de Tegucigalpa a San Pedro Sula, de Choluteca a Tegucigalpa, de San Pedro Sula a La Lima, de San Pedro Sula a Villanueva, de Danlí a Tegucigalpa, de Juticalpa a Tegucigalpa y de Santa Bárbara a San Pedro Sula.

En los períodos 1988 - 1983 y 2001 - 1996 los principales flujos migratorios recientes mayores de mil perso- 
nas se produjeron principalmente hacia Tegucigalpa, San Pedro Sula y otras ciudades intermedias como Choloma, Villanueva, La Lima y La Ceiba. En el último período específicamente los principales flujos migratorios recientes se produjeron de San Pedro Sula a Choloma, de San Pedro Sula a Villanueva y de Tegucigalpa a San Pedro Sula.

\section{MIGRACIÓN EN LAS CUATRO CIUDADES.}

Las cuatro ciudades seleccionadas justifican su estudio en varios aspectos, son las principales ciudades desde el punto de vista económico (la capital gubernamental, la primera y segunda industrial), las más pobladas (en el último censo de población del 2001, Tegucigalpa, San Pedro Sula y Choloma ocupan los primeros tres lugares, Villanueva el onceavo lugar), unas de las de mayor crecimiento demográfico intercensal, principalmente Choloma y Villanueva y desde el punto de vista migratorio, las cuatro tienen los mayores saldos migratorio positivos del país en la migración de toda la vida y reciente

\subsection{Antecedentes históricos de las cuatro ciudades.}

Tegucigalpa fue poblada por españoles que buscaban vetas de plata en el lugar, posteriormente con el crecimiento del poblado minero se le conoció con el nombre de Real Villa de San Miguel de Tegucigalpa de Heredia el 29 de septiembre de 1578 sobre un antiguo poblado indígena existente. En 1821 fue elevada al rango de ciudad. En el año 1847 se funda la primera universidad. Está ubicada en el centro del país, en el departamento de Francisco Morazán, en un altiplano, a unos $990 \mathrm{msnm}$. Rodeada de colinas, entre las que se destaca, al norte, el cerro El Picacho $(1,240 \mathrm{msnm})$. El río Choluteca cruza la ciudad de norte a sur y la divide en dos zonas: la de Tegucigalpa propiamente dicha, al este, y Comayagüela, ciudad que se fusionó con la primera en 1898, al oeste. Ambas forman el municipio del Distrito Central, como también se llama a Tegucigalpa, sede constitucional del Poder ejecutivo, Legislativo y Judicial. Es el centro político y económico de la república. Las actividades económicas más importantes de la ciudad son: la gubernamental, el comercio, construcción, servicios y otras industriales. En octubre de 1998 la ciudad sufrió daños importantes tras el paso del huracán Mitch, que destruyó una parte de Comayagüela y los lugares bordeados por el río Choluteca.

San Pedro Sula fue fundada el 27 de junio de 1536 por Pedro de Alvarado con el nombre de San Pedro de Puerto de Caballos. Ubicada en la porción oeste del Valle de Sula, en el norte del territorio nacional y en el departamento de Cortés. Su clima es húmedo de selva tropical, y sus temperaturas son muy calurosas ya que van de los 21 a los $35^{\circ} \mathrm{C}$. Se encuentra a $58 \mathrm{~km}$ al sur de Puerto Cortés (principal puerto del país, en la costa del Caribe hondureño). Sus límites son al Norte: municipio de Choloma, Sur: municipio de Villanueva, al Este: municipio de La Lima, al oeste: montaña de El Merendón y municipio de Omoa. Tiene una extensión territorial total de $838 \mathrm{~km}^{2}$. Con el auge del cultivo del banano, la llegada de las compañías trasnacionales y la construcción del ferrocarril, se dio el despegue económico y demográfico de la ciudad, que ha evolucionado muy significativamente durante la segunda mitad del siglo XX, gracias a la creación de un importante polo de desarrollo. Es la segunda ciudad más grande de Honduras, también llamada como la Capital Industrial debido al desarrollo alcanzado en el siglo XX. Es la cabecera departamental de Cortés y el principal centro urbano de la región del Valle de Sula, donde se produce aproximadamente el 55\% del Producto Interno Bruto del País.

Choloma como poblado originalmente estaba ubicado al norte del actual asentamiento y tenía el 
nombre de Tholomac, una aldea de origen precolombino de la tribu de los Xicaques. Hasta el siglo XVII el villorio fue organizado por sacerdotes franciscanos con el nombre de Santa María Candelaria, la cual sería destruida en 1739, junto a otras localidades del valle de Sula, por un alzamiento de una fuerza emancipadora contra las autoridades españolas. De esta manera, los habitantes de Candelaria fundaron Choloma, que en 1894 sería constituido como municipio bajo el nombre de El Paraíso, que después de 39 años cam-

Volumen I

$\mathbf{N}^{\circ} 2$

2010
Pág 159 industria del banano y en la siguiente centuria fue iniciado el cultivo de la caña de azúcar; sin embargo, ambas plantaciones decayeron y sus habitantes se dedicaron a la ganadería y al cultivo de cítricos y granos básicos. Otra industria que cobró auge fue la explotación de la madera. En 1974 Choloma sufrió los embates del Huracán Fifí que hizo reasentar la ciudad. A partir de 1979 se inician los beneficios de la zona libre de Puerto Cortés, pero hasta después se expanden a otros municipios (Kennedy, 1997: 9), entre ellos Choloma, constituyen el punto de arranque y despegue de la industria maquiladora en el país. En 1989 surge la zona libre de INHDELVA y ZIP Choloma donde se instalan empresas maquiladoras, de confección de ropa y diversos artículos que hace llamar al municipio como la segunda ciudad industrial de Honduras, que agrega su cercanía con Puerto Cortés por donde se realizan las exportaciones.

Villanueva fue fundado el 28 de agosto de 1871, por emigrantes del departamento de Santa Bárbara, y recibe el título de ciudad el 15 de febrero de 1945. El Municipio de Villanueva, está situado en el departamento de Cortés, a 25 kilómetros de la ciudad de San Pedro Sula. Tiene una extensión territorial de 414 $\mathrm{Km}^{2}$. El clima es tropical lluvioso, con terrenos de fuerte pendiente en un $50.7 \%$ donde predomina el bosque, el $14.5 \%$ de terrenos alomados a pendientes a cultivos permanentes y cosechas alboreas propias del clima o suelos y los restantes $34.8 \%$ son terrenos planos o suavemente ondulados con cultivos intensivos. El municipio está limitando al norte, con San Pedro Sula, al sur, con Potrerillos, San Antonio, y Concepción del Norte en Santa Bárbara; al Este con San Manuel, La Lima y Pimienta, al Oeste con Petoa, Santa Bárbara. Aunque a partir de los años noventa Villanueva comenzó a llenarse de maquilas, aumentando su movimiento comercial, la industria del azúcar sigue siendo su rubro insigne, que la hace llamarse el municipio que endulza a Honduras. Existe un número importante de maquilas, no sólo de la industria de la confección, sino de otras como artículos de cartón y plásticos, Se considera que por el cambio que tuvo Villanueva tras la llegada de la industria maquiladora, su desarrollo puede dividirse en dos etapas: antes y después de las maquilas. Aunque estas empresas no pagan impuestos al Estado por las exoneraciones, si generan cantidades de ingresos en concepto de salarios y colateralmente por la gran cantidad de servicios que los obreros demandan, en consecuencia aumenta la actividad económica del municipio.

En suma, de estas cuatro ciudades, Tegucigalpa es la capital de la república, ubicada en Francisco Morazán en el centro del país y cuna de los poderes gubernamentales. Las otras tres ubicadas en el valle de sula, cruzadas con la mejor infraestructura vial y ferrocarrilera del país, en la zona de mayor desarrollo económico, San Pedro Sula es la capital industrial del país, Choloma la segunda ciudad industrial por efectos de la maquila y junto a Villanueva son los municipios de mayor desarrollo maquilero del país. 


\subsection{Aspectos demográficos de las cuatro ciudades.}

Los departamentos de Cortés y Francisco Morazán son los más poblados de Honduras, en ellos se concentra el $36 \%$ de la población total del país y se encuentran las dos principales ciudades de la nación, Tegucigalpa y San Pedro Sula. Cortés es el departamento que más crece en Honduras, en el período intercensal de 1988 - 2001 se estima una tasa de crecimiento del 4\%. San Pedro Sula es el municipio más populoso del departamento sin embargo, hay otros como Choloma, Puerto Cortés, Villanueva, Santa Cruz de Yojoa y La Lima, que concentran un alto volumen de población. En Francisco Morazán la mayoría de la población se concentra en el municipio de Distrito Central en el cual se ubica la ciudad de Tegucigalpa.

El crecimiento acelerado de la población hondureña a partir de la segunda mitad del siglo XX ha producido tasas de crecimiento demográfico superiores al tres por ciento. Para el período intercensal 1974 1988 se registró una tasa de $3.3 \%$ mientras que para el período intercensal 1988 - 2001 se estima la misma en $2.7 \%$, considerada todavía como una de las más elevadas en el contexto latinoamericano (Flores, 2003; 6). En el caso de las cuatro ciudades de estudio el crecimiento demográfico también se manifestó, en el caso de los efectivos, de 171,146 habitantes en 1950 pasó a 2,260,601 habitantes actualmente. Tegucigalpa y San Pedro Sula ya eran consideradas ciudades de importancia en la década de los cincuenta, mientras que Choloma y Villanueva eran cabeceras municipales con escasa población y aunque todas crecieron en efectivos por el acelerado crecimiento demográfico del país, también se manifestó en estos municipios desde los años cincuenta, en el caso de los municipios maquileros este fenómeno se manifiesta más claramente en la década de los noventa (véase Gráfico No.3). En el caso de las tasas de crecimiento intercensales desde 1950 al 2001, los primeros tres municipios siempre han tenido tasas elevadas de crecimiento, mientras Villanueva fue a partir de los años setenta, incluso en el último período intercensal 1988-2001 los municipios maquileros crecen el doble que las dos principales ciudades.

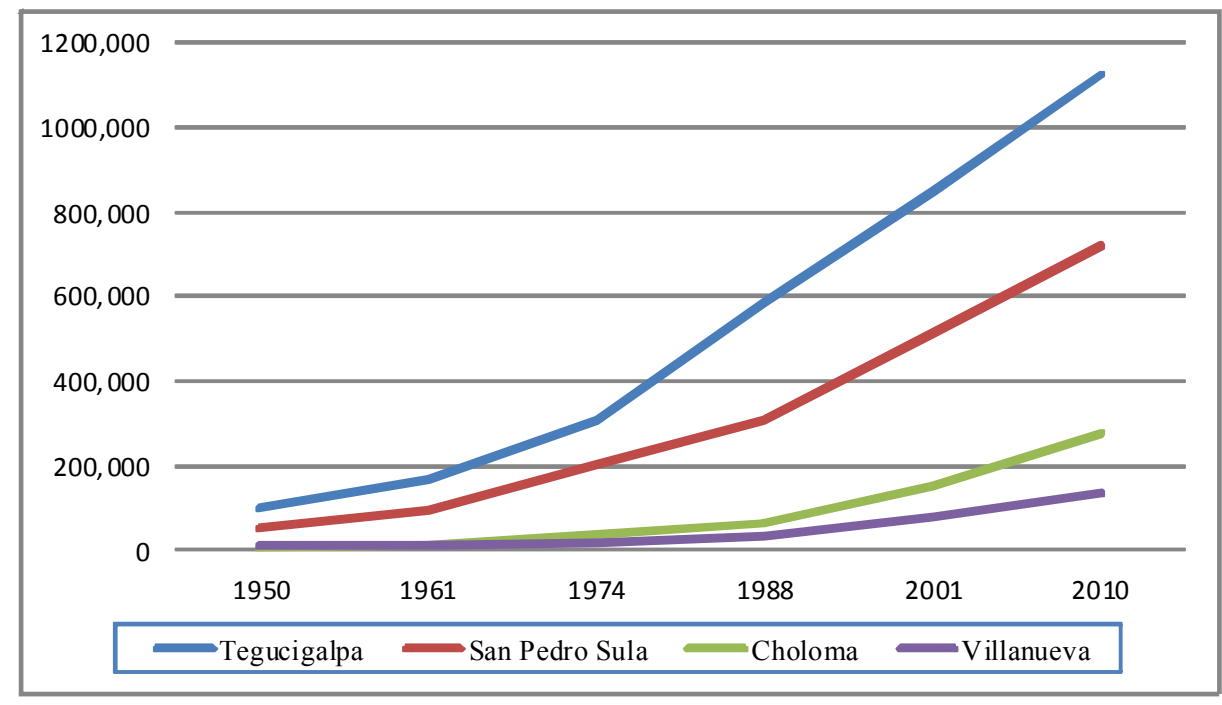

Gráfico 3. Cuatro ciudades de Honduras: Población Total, 1950-2010

Migración Interna en Cuatro Ciudades de Honduras 


\subsection{Magnitud y Cuantía Migratoria.}

En el caso del número de migrantes de toda la vida aumentó en el periodo 1988 - 2001, de 486,459 paso a 661,157 en las cuatro ciudades e incluso aumentó su proporción del total de migrantes absolutos del país, de $21.5 \%$ a $23.7 \%$. En el caso de Choloma y Villanueva el aumento superó el doble de migrantes en el pe-

Volumen I

$\mathbf{N}^{\circ} \mathbf{2}$

2010 ríodo estudiado (Cuadro No. 6).

En el caso de la migración neta, aunque el número aumentó a nivel de las cuatro ciudades en el periodo 1988 - 2001, este no fue tan elevado como en la migración bruta, sin embargo, presenta un comportamiento diferenciado, mientras en la ciudad principal el número de migrantes netos disminuye, en el caso de las ciudades de Valle de Sula aumentan los migrantes absolutos netos.

El número de inmigrantes se incrementó más que los emigrantes. Las proporciones de emigrantes e inmigrantes han descendido a nivel de tres de las ciudades en el periodo estudiado (Villanueva es la excepción en el caso de los emigrantes), sin embargo, las proporciones de inmigrantes superan a las proporciones de emigrantes en todos los municipios, principalmente en los municipios maquileros, donde el impacto inmigratorio es más significativo.

Estos resultados muestran que en la zona de estudio los migrantes absolutos aumentaron en efectivos, sin embargo sus proporciones disminuyen, en suma muestra a los municipios como áreas geográficas atractivas desde el punto de vista migratorio, principalmente en el caso de los municipios maquiladores.

En la migración reciente en los periodos 1988 - 1983 y 2001 - 1996 la migración bruta aumenta, mientras los migrantes netos disminuyen en Tegucigalpa y San Pedro Sula, presentándose el fenómeno de disminución de inmigrantes en el periodo en la ciudad capital lo que indica la pérdida de atracción de esta ciudad recientemente. Las tasas de migración neta de Choloma y Villanueva son elevadas siendo unas de las más altas en el país. Todo indica que el impacto que se produce en los municipios maquileros vecinos de San Pedro Sula es significativo y producto de la inmigración reciente (Cuadro No.7).

\subsection{Saldos Migratorios de las Cuatro Ciudades.}

Las cuatro ciudades estudiadas desde el punto de vista migratorio son atractivas, tanto en la migración absoluta como en la reciente. En el caso del intercambio migratorio de ellas con los demás DAME del país, las dos principales ciudades tienen un intercambio más diversificado, sin embargo, las maquiladoras tienen un mayor número de saldos migratorios positivos (Cuadro No.8).

Como la mayoría de los intercambios de las cuatro ciudades con el resto de municipios es positiva, revisamos los intercambios negativos. En el caso de la ciudad de Tegucigalpa en la migración de toda la vida presenta saldos migratorios negativos con las otras tres ciudades estudiadas, San Pedro Sula con Choloma, Villanueva y otras adyacentes ubicadas en el Valle de Sula. Choloma y Villanueva sus saldos migratorios negativos son insignificantes. En la migración reciente, la capital sigue el mismo patrón de saldos migratorios negativos con las otras tres ciudades estudiadas aunque en volúmenes menores. En San Pedro Sula sus saldos negativos básicamente existen por los flujos a Choloma y Villanueva. 


\begin{tabular}{|c|c|c|c|c|c|c|c|c|c|}
\hline $\begin{array}{c}\text { Municipios / } \\
\text { Ciudades }\end{array}$ & \begin{tabular}{|c} 
Población \\
residente \\
en 1988
\end{tabular} & $\begin{array}{c}\text { Población } \\
\text { nacida en el } \\
\text { municipio }\end{array}$ & $\begin{array}{c}\text { Residentes } \\
\text { nacidos en el } \\
\text { municipio }\end{array}$ & $\begin{array}{c}\text { Emigran- } \\
\text { tes }\end{array}$ & $\begin{array}{l}\text { Inmigran- } \\
\text { tes }\end{array}$ & $\begin{array}{l}\text { Migra- } \\
\text { ción } \\
\text { Neta }\end{array}$ & $\begin{array}{l}\text { Migración } \\
\text { Bruta }\end{array}$ & $\begin{array}{c}\% \\
\text { Emi- } \\
\text { grantes }\end{array}$ & $\begin{array}{c}\% \\
\text { Inmi- } \\
\text { grantes }\end{array}$ \\
\hline Tegucigalpa & 562,143 & 411,563 & 366,446 & 45,090 & 195,697 & 150,607 & 240,787 & 10.96 & 34.81 \\
\hline San Pedro Sula & 290,746 & 229,725 & 168,268 & 61,457 & 122,478 & 61,021 & 183,935 & 26.75 & 42.13 \\
\hline Choloma & 61,818 & 35,595 & 28,205 & 7,390 & 33,613 & 26,223 & 41,003 & 20.76 & 54.37 \\
\hline Villanueva & 31,870 & 22,720 & 16,928 & 5,792 & 14,942 & 9,150 & 20,734 & 25.49 & 46.88 \\
\hline Total 4 ciudades & 946,577 & 699,576 & 579,847 & 119,729 & 366,730 & 247,001 & 486,459 & 17.11 & 38.74 \\
\hline Total país & $4,112,367$ & $4,112,367$ & $2,981,903$ & $1,130,464$ & $1,130,464$ & 0 & $2,260,928$ & 27.49 & 27.49 \\
\hline $\begin{array}{c}\text { Municipios / } \\
\text { Ciudades }\end{array}$ & $\begin{array}{c}\text { Población } \\
\text { residente } \\
\text { en } 2001\end{array}$ & $\begin{array}{c}\text { Población } \\
\text { nacida en el } \\
\text { municipio }\end{array}$ & $\begin{array}{c}\text { Residentes } \\
\text { nacidos en el } \\
\text { municipio }\end{array}$ & $\begin{array}{c}\text { Emigran- } \\
\text { tes }\end{array}$ & $\begin{array}{l}\text { Inmigran- } \\
\text { tes }\end{array}$ & $\begin{array}{l}\text { Migra- } \\
\text { ción } \\
\text { Neta }\end{array}$ & $\begin{array}{c}\text { Migración } \\
\text { Bruta }\end{array}$ & $\begin{array}{c}\% \\
\text { Emi- } \\
\text { grantes }\end{array}$ & $\begin{array}{c}\% \\
\text { Inmi- } \\
\text { grantes }\end{array}$ \\
\hline Tegucigalpa & 827,941 & 687,623 & 617,225 & 70,398 & 210,716 & 140,318 & 281,114 & 10.24 & 25.45 \\
\hline San Pedro Sula & 495,539 & 402,991 & 323,219 & 79,772 & 172,320 & 92,548 & 252,092 & 19.67 & 24.19 \\
\hline Choloma & 146,055 & 81,676 & 73,206 & 8,470 & 72,849 & 64,379 & 81,319 & 10.37 & 49.88 \\
\hline Villanueva & 77,608 & 43,018 & 36,997 & 6,021 & 40,611 & 34,590 & 46,632 & 14.00 & 52.33 \\
\hline Total 4 ciudades & $1,547,143$ & $1,215,308$ & $1,050,647$ & 164,661 & 496,496 & 331,835 & 661,157 & 13.55 & 32.09 \\
\hline Total país & $5,963,794$ & $5,963,794$ & $4,112,367$ & $1,390,823$ & $1,390,823$ & 0 & $2,781,646$ & 23.32 & 23.32 \\
\hline
\end{tabular}

Cuadro 6. Cuatro ciudades de Honduras: Población y datos de migración de toda la vida, 1988 y 2001. (Fuente: Cálculos propios en base a datos censales, 1988 y 2001 )

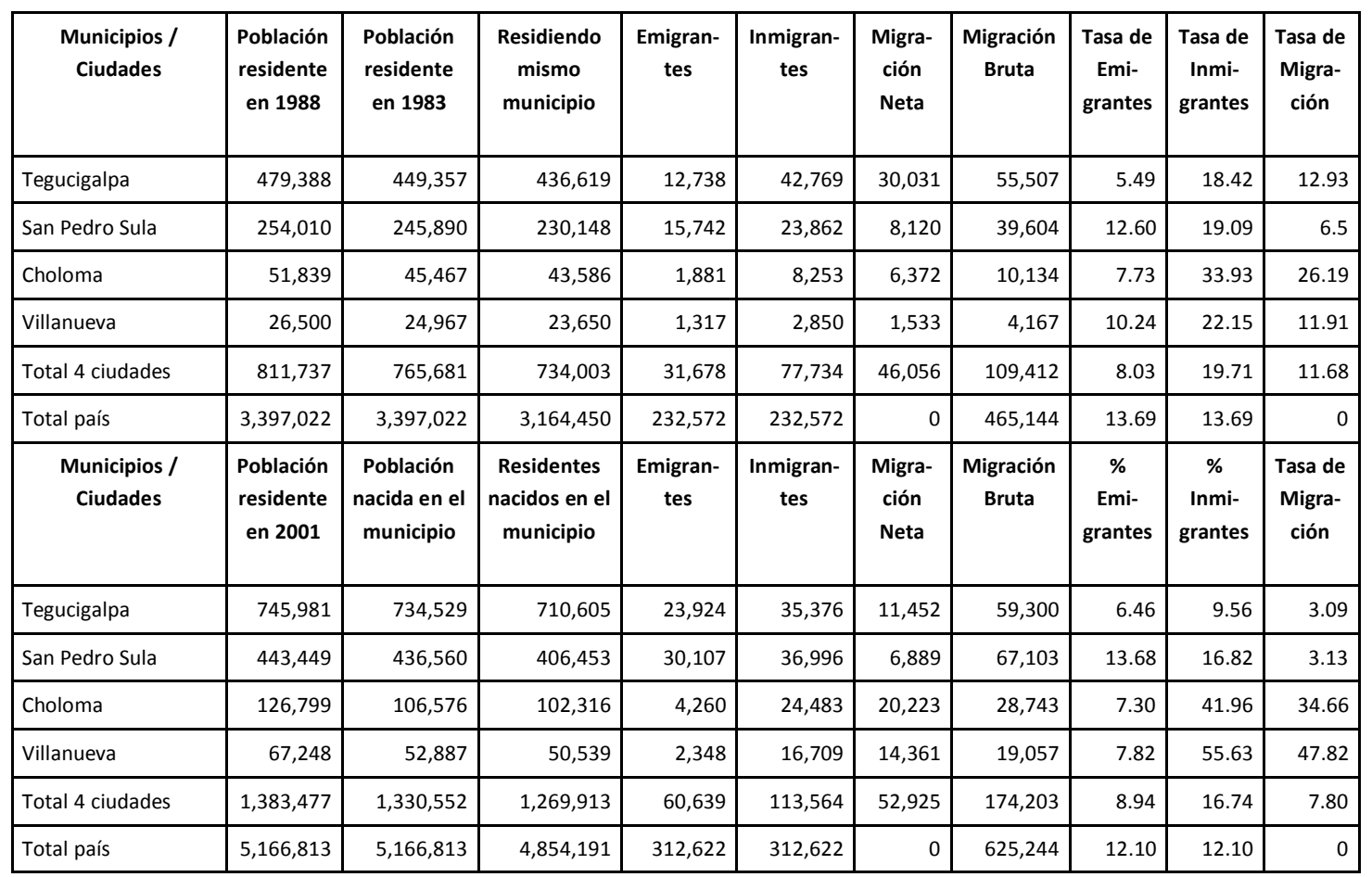

Migración Interna en Cuatro Ciudades de Honduras

Cuadro 7. Cuatro ciudades de Honduras: Población y datos de migración reciente, 1988-1983 y 2001-1996. (Fuente: Cálculos propios en base a datos censales, 1988 y 2001). 


\begin{tabular}{|c|c|c|c|c|c|c|c|}
\hline & \multirow[b]{2}{*}{ Municipios } & \multicolumn{3}{|c|}{ Migración de toda la vida DAME, 2001} & \multicolumn{3}{|c|}{ Migración Reciente DAME 2001-1996 } \\
\hline & & $\begin{array}{l}\text { Saldo Mi- } \\
\text { gratorio } \\
\text { Positivo }\end{array}$ & $\begin{array}{l}\text { Saldo Mi- } \\
\text { gratorio } \\
\text { Negativo }\end{array}$ & $\begin{array}{c}\text { Sin intercam- } \\
\text { bio Migrato- } \\
\text { rio }\end{array}$ & $\begin{array}{l}\text { Saldo Mi- } \\
\text { gratorio } \\
\text { Positivo }\end{array}$ & $\begin{array}{l}\text { Saldo Mi- } \\
\text { gratorio } \\
\text { Negativo }\end{array}$ & $\begin{array}{c}\text { Sin intercam- } \\
\text { bio Migrato- } \\
\text { rio }\end{array}$ \\
\hline Volumen I & Tegucigalpa & 273 & 24 & 0 & 225 & 61 & 0 \\
\hline $\mathbf{N}^{\circ} 2$ & San Pedro Sula & 280 & 17 & 0 & 240 & 46 & 11 \\
\hline \multirow[t]{2}{*}{2010} & Choloma & 286 & 6 & 5 & 263 & 15 & 19 \\
\hline & Villanueva & 285 & 6 & 6 & 256 & 12 & 28 \\
\hline
\end{tabular}

Cuadro 8. Cuatro ciudades de Honduras: Número de DAME por tipo de migración e intercambio migratorio según municipio, 2001. (Fuente: Cálculos propios en base a datos censales del 2001).

\subsection{Flujos Migratorios de las Cuatro Ciudades.}

En general, los más importantes flujos migratorios de toda la vida hacia las cuatro ciudades proceden de las dos principales ciudades del país en primer lugar, sumándose a ello los flujos de las otras ciudades intermedias del país, dependiendo de la ubicación, si es en el norte, principalmente es hacia las ciudades del Valle de Sula y si es centro, sur, oriente, es hacia la capital. Los mayores flujos migratorios en orden de importancia son; de San Pedro Sula a Choloma, de Tegucigalpa a San Pedro Sula, de Choluteca a Tegucigalpa, de San Pedro Sula a Villanueva, de Danlí a Tegucigalpa, de Juticalpa a Tegucigalpa, de Santa Bárbara a San Pedro Sula, de Santa Rosa de Copán a San Pedro Sula, de Pespire a Tegucigalpa y de San Pedro Sula a Tegucigalpa. Existe un fuerte intercambio entre las dos principales ciudades del país.

En la migración reciente, en general se sigue el mismo comportamiento que tiene la migración de toda la vida, aunque en menor cuantía, como era de esperarse. Los flujos entre las cuatro ciudades son muy relevantes, principalmente por los flujos a las ciudades maquileras y el intercambio entre las dos ciudades principales. Los mayores flujos de importancia se producen entre San Pedro Sula a Choloma, San Pedro Sula Villanueva, Tegucigalpa a San Pedro Sula, Danlí a Tegucigalpa, San Pedro Sula a Tegucigalpa, Choluteca a Tegucigalpa, La Lima a San Pedro Sula, Choloma a San Pedro Sula, etc.

\subsection{Migración Cercana y Lejana de las Cuatro Ciudades.}

Con la matriz de migración reciente de Honduras de 2001-1996 se construyeron las siguientes matrices por ciudad que muestran las distinciones relevantes entre los flujos para catalogarlos como migración interna cercana o lejana, tomando como base la información del municipio estudiado con respecto a otras DAME que pertenecen a la DAM respectiva u otras que están fuera de ella.

En el caso de la DAME de Tegucigalpa, esta tiene una migración neta de 11,452 personas y una tasa de migración de 3.09 por mil, esto se debe más que todo al intercambio migratorio de este municipio con otras DAME que están fuera de la DAM respectiva, aunque en ambos casos el intercambio cercano y lejano es positivo para la ciudad capital, el que más influye es el lejano (Cuadro No.9). 


\begin{tabular}{|c|c|c|c|c|c|c|}
\hline \multirow{2}{*}{$\begin{array}{l}\text { Zona de resi- } \\
\text { dencia al mo- } \\
\text { mento del cen- } \\
\text { so (2001) }\end{array}$} & \multicolumn{3}{|c|}{$\begin{array}{l}\text { Zona de residencia cinco años antes del cen- } \\
\text { so (1996) }\end{array}$} & \multirow[b]{2}{*}{$\begin{array}{l}\text { Total Cen- } \\
\text { so }\end{array}$} & \multirow{2}{*}{$\begin{array}{l}\text { Migración } \\
\text { neta (total } \\
\text { censo- } \\
\text { total cinco } \\
\text { años antes) }\end{array}$} & \multirow{2}{*}{$\begin{array}{c}\text { Tasa de } \\
\text { migra- } \\
\text { ción } \\
\text { neta } \\
\text { (por mil) }\end{array}$} \\
\hline & Tegucigalpa & $\begin{array}{l}\text { Otro munici- } \\
\text { pio de Francis- } \\
\text { co Morazán }\end{array}$ & $\begin{array}{l}\text { Otro municipio } \\
\text { fuera de Fran- } \\
\text { cisco Morazán }\end{array}$ & & & \\
\hline Tegucigalpa & 710,605 & 5,704 & 29,672 & 745,981 & 11,452 & 3.09 \\
\hline $\begin{array}{l}\text { Otro municipio } \\
\text { de Francisco } \\
\text { Morazán }\end{array}$ & 4,518 & 212,919 & 4,566 & 222,003 & & \\
\hline $\begin{array}{l}\text { Otro municipio } \\
\text { fuera de Fran- } \\
\text { cisco Morazán }\end{array}$ & 19,406 & 3,956 & $4,175,467$ & $4,198,829$ & & \\
\hline Total & 734,529 & 222,579 & $4,209,705$ & $5,166,813$ & & \\
\hline
\end{tabular}

Cuadro 9. Tegucigalpa: Matriz de Migración Reciente con otros municipios del país agrupados en categorías de cercanía, 2001-1996. (Fuente: Cálculos propios de la Matriz Básica de Migración Reciente, MIALC-CELADE)

En San Pedro Sula como centro industrial del norte del país, los datos indican una migración neta positiva de 6,889 personas y una tasa de migración neta de 3.13 por mil, aunque esta se debe exclusivamente al intercambio migratorio del municipio con los otros municipios localizados fuera de la DAM respectiva, mientras que la migración neta con el resto de municipio de la misma DAM es negativo, es decir que la ciudad mantiene un alto atractivo migratorio con el resto del país, pero es negativo con las otras entidades de su misma DAM, todo esto vislumbra un panorama que podría explicarse por la ampliación de los límites de la ciudad hacía los municipios adyacentes, entre los cuales están las ciudades de Choloma y Villanueva, donde a raíz de la ampliación del sector maquilador atraen población de San Pedro Sula e incluso hay un crecimiento de la infraestructura habitacional que influye en el comportamiento migratorio (Cuadro No.10).

En Choloma, el comportamiento de la migración reciente muestra como la ciudad presenta una migración neta positiva de 20,223 y una tasa de migración neta 34.66 por mil, superior a las dos ciudades principales. Esta migración neta se debe a los intercambios cercanos y lejanos porque con ambos casos es positivo (Cuadro No.11).

Villanueva como municipio de atracción migratoria más reciente por su transformación industrial que ha tenido lugar hace pocos años con la llegada masiva de empresas maquiladoras, el impacto demográfico es más notable en su tasa de migración neta que alcanza el 47.82 por mil, siendo la más elevada de las DAME del país, que se debe a intercambios cercanos y lejanos (Cuadro No.12).

En suma, en las cuatro ciudades los niveles de migración neta son positivos, principalmente porque las diferencias migratorias lejanas son mayormente positivas que las cercanas. El caso de San Pedro Sula merece atención por su intercambio migratorio cercano negativo que se produce principalmente por saldos migratorios negativos con Villanueva y Choloma, ciudades adyacentes, que resultan atractivas por el empleo maquilero y otras actividades económicas, que incide para cambiar de residencia (Gráfico No.4). 
Volumen I

$N^{\circ} 2$

2010

\begin{tabular}{|c|c|c|c|c|c|c|}
\hline \multirow{2}{*}{$\begin{array}{l}\text { Zona de resi- } \\
\text { dencia al mo- } \\
\text { mento del } \\
\text { censo (2001) }\end{array}$} & \multicolumn{3}{|c|}{$\begin{array}{l}\text { Zona de residencia cinco años antes del censo } \\
\qquad(1996)\end{array}$} & \multirow{2}{*}{$\begin{array}{l}\text { Total Cen- } \\
\text { so }\end{array}$} & \multirow{2}{*}{$\begin{array}{l}\text { Migración } \\
\text { neta (total } \\
\text { censo- } \\
\text { total cinco } \\
\text { años antes) }\end{array}$} & \multirow{2}{*}{$\begin{array}{c}\text { Tasa de } \\
\text { migra- } \\
\text { ción neta } \\
\text { (por mil) }\end{array}$} \\
\hline & $\begin{array}{l}\text { San Pedro } \\
\text { Sula }\end{array}$ & $\begin{array}{l}\text { Otro munici- } \\
\text { pio de Cortés }\end{array}$ & $\begin{array}{l}\text { Otro municipio } \\
\text { fuera de Cortés }\end{array}$ & & & \\
\hline San pedro sula & 406,453 & 5,122 & 31,874 & 443,449 & 6,889 & 3.13 \\
\hline $\begin{array}{l}\text { Otro munici- } \\
\text { pio de Cortés }\end{array}$ & 16,603 & 417,579 & 40,865 & 475,047 & & \\
\hline $\begin{array}{l}\text { Otro munici- } \\
\text { pio fuera de } \\
\text { Cortés }\end{array}$ & 13,504 & 7,855 & $4,226,958$ & $4,248,317$ & & \\
\hline Total & 436,560 & 430,556 & $4,299,697$ & $5,166,813$ & & \\
\hline
\end{tabular}

Cuadro 10. San Pedro Sula: Matriz de migración reciente con otros municipios del país agrupados en categorías de cercanía, 2001-1996. (Fuente: Cálculos propios de la Matriz básica de migración reciente, MIALC-CELADE).

\begin{tabular}{|c|c|c|c|c|c|c|}
\hline \multirow{2}{*}{$\begin{array}{l}\text { Zona de resi- } \\
\text { dencia al mo- } \\
\text { mento del } \\
\text { censo (2001) }\end{array}$} & \multicolumn{3}{|c|}{$\begin{array}{l}\text { Zona de residencia cinco años antes del censo } \\
\qquad(1996)\end{array}$} & \multirow{2}{*}{ Total Censo } & \multirow{2}{*}{$\begin{array}{c}\text { Migración } \\
\text { neta (total } \\
\text { censo- } \\
\text { total cinco } \\
\text { años antes) }\end{array}$} & \multirow{2}{*}{$\begin{array}{l}\text { Tasa de } \\
\text { migra- } \\
\text { ción neta } \\
\text { (por mil) }\end{array}$} \\
\hline & Choloma & $\begin{array}{l}\text { Otro munici- } \\
\text { pio de Cortés }\end{array}$ & $\begin{array}{l}\text { Otro municipio } \\
\text { fuera de Cortés }\end{array}$ & & & \\
\hline Choloma & 102,316 & 7,776 & 16,707 & 126,799 & 20,223 & 34.66 \\
\hline $\begin{array}{l}\text { Otro munici- } \\
\text { pio de Cortés }\end{array}$ & 2,458 & 733,207 & 56,032 & 791,697 & & \\
\hline $\begin{array}{l}\text { Otro munici- } \\
\text { pio fuera de } \\
\text { Cortés }\end{array}$ & 1,802 & 19,557 & $4,226,958$ & $4,248,317$ & & \\
\hline Total & 106,576 & 760,540 & 4,299,697 & $5,166,813$ & & \\
\hline
\end{tabular}

Cuadro 11. Choloma: Matriz de migración reciente con otros municipios del país agrupados en categorías de cercanía, 2001-1996. (Fuente: Cálculos propios de la Matriz básica de migración reciente, MIALC-CELADE). 


\begin{tabular}{|c|c|c|c|c|c|c|c|}
\hline \multirow{2}{*}{$\begin{array}{l}\text { Zona de resi- } \\
\text { dencia al mo- } \\
\text { mento del } \\
\text { censo (2001) }\end{array}$} & \multicolumn{3}{|c|}{$\begin{array}{l}\text { Zona de residencia cinco años antes del censo } \\
\qquad(1996)\end{array}$} & \multirow{2}{*}{$\begin{array}{l}\text { Total Cen- } \\
\text { so }\end{array}$} & \multirow{2}{*}{$\begin{array}{l}\text { Migración } \\
\text { neta (total } \\
\text { censo- } \\
\text { total cinco } \\
\text { años antes) }\end{array}$} & \multirow{2}{*}{$\begin{array}{c}\text { Tasa de } \\
\text { migra- } \\
\text { ción neta } \\
\text { (por mil) }\end{array}$} & \multirow{6}{*}{$\begin{array}{l}\text { Migración } \\
\text { Interna en } \\
\text { Cuatro Ciudades } \\
\text { de Honduras }\end{array}$} \\
\hline & Villanueva & $\begin{array}{l}\text { Otro munici- } \\
\text { pio de Cortés }\end{array}$ & $\begin{array}{l}\text { Otro municipio } \\
\text { fuera de Cortés }\end{array}$ & & & & \\
\hline Villanueva & 50,539 & 7,530 & 9,179 & 67,248 & 14,361 & 47.82 & \\
\hline $\begin{array}{l}\text { Otro munici- } \\
\text { pio de Cortés }\end{array}$ & 1,632 & 786,056 & 63,560 & 851,248 & & & \\
\hline $\begin{array}{l}\text { Otro munici- } \\
\text { pio fuera de } \\
\text { Cortés }\end{array}$ & 716 & 20,643 & $4,226,958$ & $4,248,317$ & & & \\
\hline Total & 52,887 & 814,229 & $4,299,697$ & $5,166,813$ & & & \\
\hline
\end{tabular}

Cuadro 12. Villanueva: Matriz de migración reciente con otros municipios del país agrupados en categorías de cercanía, 2001-1996. (Fuente: Cálculos propios de la Matriz básica de migración reciente, MIALC-CELADE).

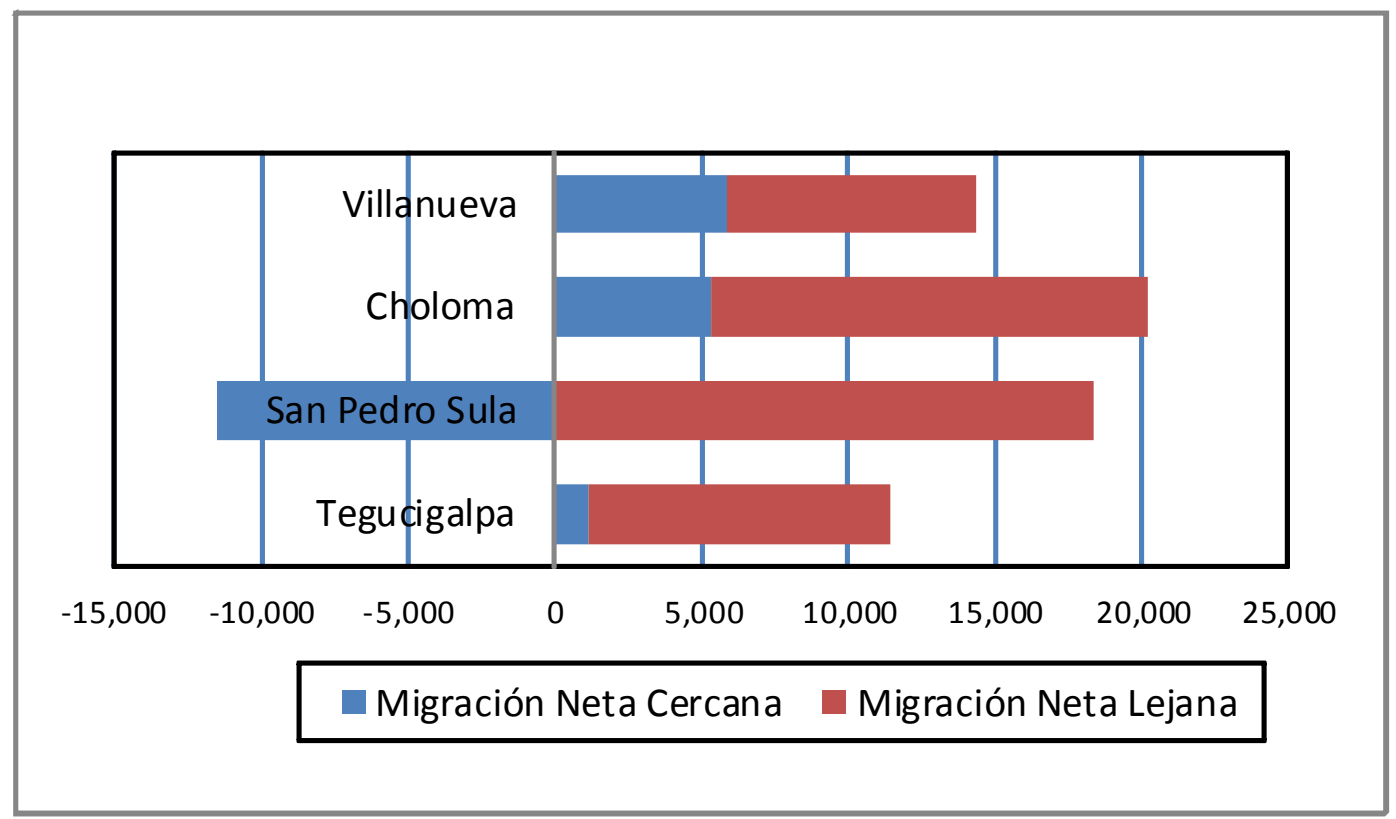

Gráfico 4. Cuatro ciudades de Honduras: Migración neta cercana y lejana. 


\section{A MANERA DE CONCLUSIÓN.}

Estas cuatro ciudades registraron los mayores volúmenes de inmigrantes y los más elevados saldos migratorios positivos a nivel de DAME en el último censo de población del 2001, tanto en la migración

Volumen I

$\mathbf{N}^{\circ} 2$

2010 de toda la vida y migración reciente, donde incluso en esta última los municipios maquiladores adquirieron supremacía nacional con respecto a las dos ciudades más pobladas y principales del país.

El municipio de Villanueva tiene la tasa de migración neta reciente más elevada del país, la población se incrementó en cuarenta y ocho personas por mil por el efecto de la migración en la última estimación censal de la migración reciente, esto por el empuje de las industrias maquiladoras y otras industrias.

En general, las ciudades en estudio mantienen su atractivo migratorio reciente y la cuantía desde el resto del país continúa siendo una fuerza importante. En las cuatro ciudades la migración interna neta positiva se debe exclusivamente al intercambio migratorio de ellas con otros municipios ubicados en otras DAM del resto de Honduras, ya que la migración neta con otros municipios de su DAM es menor. En el caso de San Pedro Sula el saldo migratorio interno positivo resulta de la diferencia que es mayor de la combinación del intercambio con los municipios pertenecientes a las otras DAM y el saldo interno negativo en el intercambio con el resto de municipios de su propia DAM, con lo que este municipio tiene la tendencia de pérdida de atractivo en su DAM producto posiblemente a la ampliación de sus límites a los municipios adyacentes, que incluye a varios municipios de la DAM (entre ellos Choloma y Villanueva) y otros municipios que aunque vecinos que pertenecen a otra DAM. 


\section{REFERENCIAS}

FLORES, Manuel (2003), Estado de la Población de Honduras, Tegucigalpa, Instituto de Investigaciones Económicas y Sociales (IIES), Universidad Nacional Autónoma de Honduras (UNAH).

FLORES, Manuel (2005), Migración interna intermunicipal de Honduras, Tegucigalpa, Instituto de Investigaciones Económicas y Sociales (IIES), Universidad Nacional Autónoma de Honduras (UNAH).

FLORES, Manuel (2006), Estimaciones de migración rural - urbana, Tegucigalpa, Instituto de Investigaciones Económicas y Sociales (IIES), Universidad Nacional Autónoma de Honduras (UNAH).

FLORES, Manuel (2009), La Medición Censal de la Migración en Honduras, Notas de Población, Año XXXVI, 88, Santiago de Chile, Comisión Económica para América Latina y el Caribe (CEPAL).

KENNEDY, Mirta (1997), Mujeres y Maquilas en Honduras, Tegucigalpa, Centro de Estudios de la Mujer.

RODRIGUEZ, Jorge (2004), Migración interna en América Latina y El Caribe: estudio regional del período 1980-2000, serie Población y desarrollo, 50, Santiago de Chile, Comisión Económica para América Latina y el Caribe (CEPAL).

RODRIGUEZ, Jorge y Busso, Gustavo (2009). Migración Interna y Desarrollo en América Latina entre 1980 y 2005. Un estudio comparativo con perspectiva regional basado en siete países. Santiago de Chile, Comisión Económica para América Latina y el Caribe (CEPAL). 